\title{
GROTHENDIECK GROUPS FOR HYPERSURFACE RINGS
}

\author{
ØYVIND SOLBERG
}

(Communicated by Maurice Auslander)

\begin{abstract}
Let $R$ and $S$ be commutative complete noetherian local Gorenstein domains. If the category of finitely generated maximal Cohen-Macaulay modules over $R$ and $S$ are stably equivalent and the equivalence commutes with the first syzygy functors, then we show that the Grothendieck groups for $R$ and $S$ are isomorphic. In particular, we apply this result to hypersurface rings.
\end{abstract}

\section{INTRODUCTION}

Let $R$ and $S$ be complete commutative local noetherian Gorenstein rings and $\Lambda$ and $\Lambda^{\prime}$ algebras over $R$ and $S$ respectively such that $\Lambda$ and $\Lambda^{\prime}$ are finitely generated Cohen-Macaulay modules over $R$ and $S$, respectively. We denote by $\mathrm{CM}_{R}(\Lambda)$ the category of all finitely generated $\Lambda$-modules which are Cohen-Macaulay modules over $R$. If $\underline{\mathrm{CM}}_{R}(\Lambda)$ denotes the category $\mathrm{CM}_{R}(\Lambda)$ modulo all finitely generated projective $\Lambda$-modules and there exists an equivalence $\alpha: \underline{\mathrm{CM}}_{R}(\Lambda) \rightarrow \underline{\mathrm{CM}}_{S}\left(\Lambda^{\prime}\right)$ that commutes with the first syzygy, we show that the stable Grothendieck groups are isomorphic (see $\S 1$ for definition). In particular we apply this to selfinjective artinian algebras over commutative local (Gorenstein) rings and to commutative complete noetherian local Gorenstein domains. These results are shown by using functor categories and using results from [2-5].

The main motivation for this work was the hypersurface case. Let $T=$ $k\left[\left[z_{1}, \ldots, z_{r}\right]\right]$ be the formal power series ring in the indeterminates $z_{i}$ over an algebraically closed field $k$ of characteristic different from 2 . If $f$ is a nonzero nonunit in $T$, then it is shown in [8] that the stable categories of Cohen-Macaulay modules over $R=T /(f)$ and $S=T[[x, y]] /(f+x y)$ are equivalent and the equivalence commutes with the first syzygy. If $R$ is a domain it follows from [3, Chapter 3, Proposition 1.1] that the Grothendieck group for

Received by the editors October 6, 1989 and, in revised form, December 5, 1989.

1980 Mathematics Subject Classification (1985 Revision). Primary 18F30, 13D15, 16A54; Secondary $13 \mathrm{H} 10,16 \mathrm{~A} 35$.

This paper was written while the author was supported by the NAVF (Norwegian Research Council). 
$R$ is determined by the stable Grothendieck group of the category of CohenMacaulay modules over $R$. So, by using the general result mentioned above, we show that the Grothendieck groups for $R$ and $S$ are isomorphic. This result was known when $R$ is a domain of finite Cohen-Macaulay type because of the following additional facts. The ring $R$ is of finite Cohen-Macaulay type if and only if $S$ is [8]. If $R$ is a domain of finite Cohen-Macaulay type, then the Grothendieck group of $R$ is determined by the stable AR-quiver for the category of Cohen-Macaulay modules over $R$ [3, Chapter 2, Proposition 2.2 and Chapter 3, Proposition 1.1 (b)].

We end the paper by giving an example showing that we cannot remove the assumption that both rings are domains.

\section{BASIC RESULTS}

In this section we recall definitions and results from [2-5] that will be used to prove our main result. Further, we show a result about stable Grothendieck groups for not necessarily commutative rings, that we apply to stably equivalent selfinjective artinian algebras over commutative local (Gorenstein) rings.

Let $R$ be a complete commutative local noetherian Gorenstein ring, $\Lambda$ an $R$-algebra that is a finitely generated Cohen-Macaulay module over $R$, and let $\mathrm{CM}_{R}(\Lambda)$ denote the category of all finitely generated $\Lambda$-modules that are Cohen-Macaulay modules over $R$. For an additive category $\mathscr{C}$ denote by $\operatorname{Mod} \mathscr{C}$ the category of additive functors from $\mathscr{C}^{\text {op }}$ to the category of abelian groups and by $\bmod \mathscr{C}$ the category of finitely presented functors $\operatorname{Mod} \mathscr{C}$, that is, functors $F \in \operatorname{Mod} \mathscr{C}$ which have a presentation $(, A) \stackrel{(, f)}{\rightarrow}(, B) \rightarrow F \rightarrow 0$ for some $f: A \rightarrow B$ in $\mathscr{C}$. If $\mathscr{P}(\Lambda)$ denotes the category of finitely generated projective $\Lambda$-modules, we denote by $\mathrm{CM}_{R}(\Lambda) / \mathscr{P}(\Lambda)=\mathrm{CM}_{R}(\Lambda)$ the category $\mathrm{CM}_{R}(\Lambda)$ modulo the finitely generated projective $\Lambda$-modules. In mod $\mathrm{CM}_{R}(\Lambda)$ let $(, \underline{A})$ denote $\operatorname{Hom}_{\Lambda}(, A) / P(, A)$, where $P(X, A)$ is all the $\Lambda$-homomorphisms from $X$ to $A$ that factor through a projective $\Lambda$-module. Then, observing that the proofs in $[2,4,5]$ work with only cosmetic changes, the following results are still true in our setting, where projective and injective always mean Ext-projective and Ext-injective, respectively.

Proposition 1 [2,5]. (a) All the projective objects in $\bmod \underline{C M}_{R}(\Lambda)$ are of the form $(, \underline{A})$ for some $A$ in $\underline{\mathrm{CM}}_{R}(\Lambda)$.

(b) All the injective objects in $\bmod \mathrm{CM}_{R}(\Lambda)$ are of the form $\operatorname{Ext}_{\Lambda}^{1}(, A)$ for some $A$ in $\underline{\mathrm{CM}}_{R}(\Lambda)$.

(c) If $0 \rightarrow A \stackrel{f}{\rightarrow} B \stackrel{g}{\rightarrow} C \rightarrow 0$ is an exact sequence in $\mathrm{CM}_{R}(\Lambda)$ without split exact summands, then we have

(i) $0 \rightarrow(, A) \stackrel{(, f)}{\rightarrow}(, B) \stackrel{(, g)}{\rightarrow}(, C) \rightarrow F \rightarrow 0$ is a minimal projective resolution of $F=\operatorname{coker}(, g)$ in $\bmod \mathrm{CM}_{R}(\Lambda)$.

(ii) $(, A) \stackrel{(, f)}{\rightarrow}(, B) \stackrel{(, g)}{\rightarrow}(, \underline{C}) \rightarrow F \rightarrow 0$ is the start of a projective resolution of $F$ in $\bmod \underline{C M}_{R}(\Lambda)$ which is minimal in the first two steps. 
(iii) $0 \rightarrow F \rightarrow \operatorname{Ext}_{\Lambda}^{1}(, A) \rightarrow \operatorname{Ext}_{\Lambda}^{1}(, B)$ is the start of a minimal injective resolution of $F$ in $\bmod \underline{C M}_{R}(\Lambda)$.

Theorem 2 [4]. Let $A, B$, and $C$ be in $\mathrm{CM}_{R}(\Lambda)$ without any nonzero projective summands, where $A$ is indecomposable.

(a) Let $0 \rightarrow A \stackrel{f}{\rightarrow} B \oplus P \stackrel{g}{\rightarrow} C \rightarrow 0$ be an exact sequence without split exact summands in $\mathrm{CM}_{R}(\Lambda)$, where $P$ is in $\mathscr{P}(\Lambda)$ and $\underline{f} \neq 0$ in $\underline{\operatorname{Hom}}_{\Lambda}(A, B)$. Then,

$$
(, \underline{A}) \stackrel{(f)}{\rightarrow}(, \underline{B}) \stackrel{(, g}{\rightarrow}(, \underline{C}) \rightarrow F \rightarrow 0
$$

is the start of a minimal projective resolution of $F=\operatorname{coker}(, \underline{g})$ in $\bmod \underline{C M}_{R}(\Lambda)$ and the injective envelope of $F$ is indecomposable.

(b) Let $(, \underline{A}) \stackrel{\mu}{\rightarrow}(, \underline{B}) \stackrel{\nu}{\rightarrow}(, \underline{C}) \rightarrow F \rightarrow 0$ be the start of a minimal projective resolution of $F=\operatorname{coker} \nu$ in $\bmod \mathrm{CM}_{R}(\Lambda)$, where the injective envelope of $F$ is indecomposable. Then there exists an exact sequence

$$
0 \rightarrow A \stackrel{f}{\rightarrow} B \oplus P \stackrel{g}{\rightarrow} C \rightarrow 0
$$

without split exact summands in $\mathrm{CM}_{R}(\Lambda)$, where $P$ is in $\mathscr{P}(\Lambda)$ such that $\mu=(, \underline{f})$ and $\nu=(, \underline{g})$.

Proposition 3 [4]. Let $S$ and $\Lambda^{\prime}$ satisfy the same assumptions as $R$ and $\Lambda$. Let $\alpha: \underline{\mathrm{CM}}_{R}(\Lambda) \rightarrow \underline{\mathrm{CM}}_{S}\left(\Lambda^{\prime}\right)$ be an equivalence, $0 \rightarrow A \stackrel{f}{\rightarrow} B \oplus P \stackrel{g}{\rightarrow} C \rightarrow 0$ an exact sequence in $\mathrm{CM}_{R}(\Lambda)$ with no split exact summand, $A, B$, and $C$ without any nonzero projective summands and $P$ projective. Assume that $A$ is indecomposable and $\underline{f}$ is not zero in $\underline{\operatorname{Hom}}_{\Lambda}(A, B)$. Then there is an exact sequence $0 \rightarrow \alpha(A) \stackrel{f^{\prime}}{\rightarrow} \alpha(B) \oplus Q \stackrel{g^{\prime}}{\rightarrow} \alpha(C) \rightarrow 0$ in $\mathrm{CM}_{S}\left(\Lambda^{\prime}\right)$, with no split exact summands, where $Q$ is projective, $\alpha(\underline{f})=\underline{f}^{\prime}$ and $\alpha(\underline{g})=\underline{g}^{\prime}$.

Let $K_{0}(\bmod \Lambda)$ be the free abelian group on all isomorphism classes of modules in $\bmod \Lambda$ modulo the relations given by all the exact sequences in $\bmod \Lambda$. For a module $A$ in $\bmod \Lambda$ we denote by $[A]$ the corresponding element in the free abelian group on all isomorphism classes of modules in $\bmod \Lambda$. It is well known that the Grothendieck group $K_{0}(\bmod \Lambda)$ is isomorphic to $K_{0}\left(\mathrm{CM}_{R}(\Lambda)\right)$. If in addition $\Lambda$ is a local ring, there is an exact sequence of groups $0 \rightarrow \mathbf{Z} \rightarrow K_{0}\left(\mathrm{CM}_{R}(\Lambda)\right) \rightarrow \underline{K}_{0}\left(\underline{\mathrm{CM}}_{R}(\Lambda)\right) \rightarrow 0$, and if $\Lambda$ is local domain, the sequence splits [3, Chapter 3]. Here, $\underline{K}_{0}\left(\mathrm{CM}_{R}(\Lambda)\right)$ is the free abelian group on all isomorphism classes of modules in $\mathrm{CM}_{R}(\Lambda)$ without any nonzero projective summands, modulo the relations $[A]+[C]-[B]$, if there exists an exact sequence $0 \rightarrow A \oplus P_{1} \rightarrow B \oplus P_{2} \rightarrow C \oplus P_{3} \rightarrow 0$ in $\mathrm{CM}_{R}(\Lambda)$ with $P_{i}$ in $\mathscr{P}(\Lambda)$ for $i=1,2,3$. Let $\Lambda$ and $\Lambda^{\prime}$ be algebras over $R$ and $S$ respectively, where $R$ and $S$ are arbitrary complete commutative local noetherian Gorenstein rings, such that $\Lambda\left(\Lambda^{\prime}\right)$ is a (maximal) Cohen-Macaulay $R$-module $(S$-module $)$ and $\operatorname{Hom}_{R}\left(\Lambda^{\mathrm{op}}, R\right) \quad\left(\operatorname{Hom}_{S}\left(\left(\Lambda^{\prime}\right)^{\mathrm{op}}, S\right)\right)$ is a projective $\Lambda$-module $\left(\Lambda^{\prime}\right.$-module). In this setting, we show that $\underline{K}_{0}\left(\underline{\mathrm{CM}}_{R}(\Lambda)\right) \simeq K_{0}\left(\underline{\mathrm{CM}}_{S}\left(\Lambda^{\prime}\right)\right)$ if $\underline{\mathrm{CM}}_{R}(\Lambda)$ and $\underline{\mathrm{CM}}_{S}\left(\Lambda^{\prime}\right)$ are equivalent and the equivalence "commutes" with 
the syzygy functors. Our assumptions hold for Gorenstein $R$-orders, where $R$ is as above. We recall that for an equidimensional Gorenstein $\operatorname{ring} T$ a noetherian $T$-algebra $\Gamma$ is said to be a Gorenstein $T$-order if $\Gamma$ is a (maximal) Cohen-Macaulay $T$-module and if $\Gamma$ is isomorphic to $\operatorname{Hom}_{T}(\Gamma, T)$ as two-sided $\Gamma$-modules [1].

In the proof of the above-mentioned result we need that $\bmod \underline{C M}_{R}(\Lambda)$, like the category $\mathrm{CM}(R)=\mathrm{CM}_{R}(R)$, has the property that the injective and projective objects coincide.

Lemma 4. Let $R$ and $\Lambda$ be as above. Then the injective and projective objects in $\bmod \underline{\mathrm{CM}}_{R}(\Lambda)$ coincide.

Proof. Since $R$ is Gorenstein, $D=\operatorname{Hom}_{R}(, R)$ is a duality on the category of Cohen-Macaulay modules over $R$, and therefore, $D: \mathrm{CM}_{R}(\Lambda) \rightarrow \mathrm{CM}_{R}\left(\Lambda^{\mathrm{op}}\right)$ is also a duality. Since ${ }_{\Lambda} \Lambda \simeq{ }_{\Lambda} D\left(\Lambda^{\mathrm{op}}\right)$, all injective modules in $\mathrm{CM}_{R}(\Lambda)$ are also projective. For $A$ in $\mathrm{CM}_{R}(\Lambda)$ without any nonzero injective summands we have the following exact sequence in $\mathrm{CM}_{R}(\Lambda)$ without split exact summands, $0 \rightarrow A \rightarrow I \rightarrow \Omega^{-1} A \rightarrow 0$, where $I$ is injective (and projective) in $\operatorname{CM}_{R}(\Lambda)$. It follows from Proposition 1 that we have the following exact sequence.

$$
(, \underline{I}) \rightarrow\left(, \underline{\Omega^{-1} A}\right) \rightarrow \operatorname{Ext}_{\Lambda}^{1}(, A) \rightarrow \operatorname{Ext}_{\Lambda}^{1}(, I) .
$$

Since both end terms of this sequence are zero, $\left(, \underline{\Omega^{-1} A}\right) \simeq \operatorname{Ext}_{\Lambda}^{1}(, A)$ and similarly $(, \underline{A}) \simeq \operatorname{Ext}_{\Lambda}^{1}(, \Omega A)$, hence the injective and projective objects in $\bmod \underline{\mathrm{CM}}_{R}(\Lambda)$ coincide.

Let $\Lambda$ and $\Lambda^{\prime}$ be algebras over $R$ and $S$ respectively, where $R$ and $S$ are arbitrary, complete, commutative, local, noetherian Gorenstein rings, such that $\Lambda\left(\Lambda^{\prime}\right)$ is a (maximal) Cohen-Macaulay $R$-module ( $S$-module) and $\operatorname{Hom}_{R}\left(\Lambda^{\text {op }}, R\right)\left(\operatorname{Hom}_{S}\left(\left(\Lambda^{\prime}\right)^{\text {op }}, S\right)\right)$ is a projective $\Lambda$-module $\left(\Lambda^{\prime}\right.$-module $)$. Let $\Omega_{\Lambda}\left(\Omega_{\Lambda}^{\prime}\right)$ denote the first syzygy in $\bmod \Lambda\left(\bmod \Lambda^{\prime}\right)$.

Theorem 5. Let $\Lambda, \Lambda^{\prime}, R$, and $S$ be as above. If there exists an equivalence $\alpha: \underline{\mathrm{CM}}_{R}(\Lambda) \rightarrow \underline{\mathrm{CM}}_{S}\left(\Lambda^{\prime}\right)$ such that $\Omega_{\Lambda^{\prime}} \circ \alpha \simeq \alpha \circ \Omega_{\Lambda}$ on objects, then

$$
\underline{K}_{0}\left(\underline{\mathrm{CM}}_{R}(\Lambda)\right) \simeq \underline{K}_{0}\left(\underline{\mathrm{CM}}_{S}\left(\Lambda^{\prime}\right)\right)
$$

Proof. In the following way we choose a particular set of generators for the relations in $\underline{K}_{0}\left(\underline{\mathrm{CM}}_{R}(\Lambda)\right)$. If $[A]+[C]-[B]$ is a relation in $\underline{K}_{0}\left(\underline{\mathrm{CM}}_{R}(\Lambda)\right)$, then there exists an exact sequence $0 \rightarrow A \oplus P_{1} \rightarrow B \oplus P_{2} \rightarrow C \oplus P_{3} \rightarrow 0$, where the $P_{i}$ are projective in $\mathrm{CM}_{R}(\Lambda)$ for $i=1,2,3$. Suppose that $A \simeq A_{1} \oplus A_{2}$. 
Then we have the following diagram in $\operatorname{CM}_{R}(\Lambda)$.

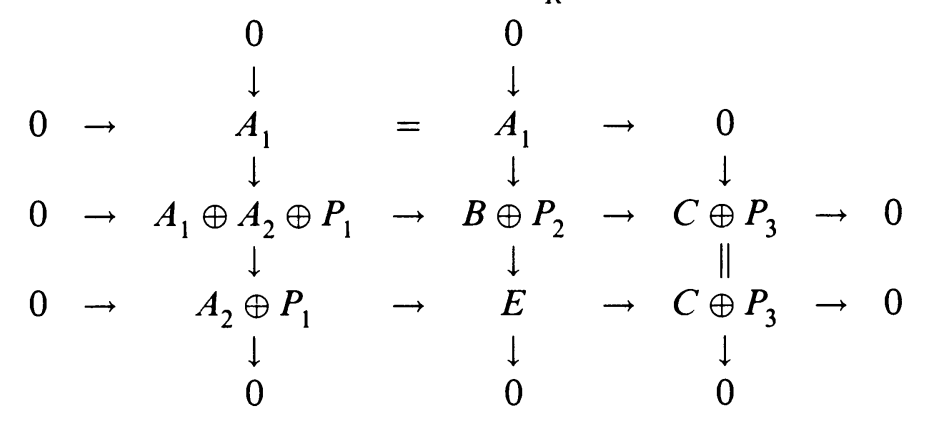

It follows that $[A]+[C]-[B]=\left(\left[A_{1}\right]+[E]-[B]\right)+\left(\left[A_{2}\right]+[C]-[E]\right)$, hence every relation is a sum of elements of the form $[A]+[C]-[B]$, where $A$ is indecomposable, and there exists an exact sequence $0 \rightarrow A \oplus P_{1} \rightarrow B \oplus P_{2} \rightarrow$ $C \oplus P_{3} \rightarrow 0$ with $P_{i}$ projective for $i=1,2,3$. Since $\Lambda$ is injective (and projective) in $\mathrm{CM}_{R}(\Lambda), P_{1}$ and $P_{3}$ split off, so that every relation is a sum of elements of the form $[A]+[C]-[B]$, where $A$ is indecomposable, $A, B$, and $C$ without any nonzero projective summands and there exists an exact sequence $0 \rightarrow A \rightarrow B \oplus P \rightarrow C \rightarrow 0$ in $\mathrm{CM}_{R}(\Lambda)$ with $P$ projective.

Define $\tilde{\alpha}: \underline{K}_{0}\left(\underline{\mathrm{CM}}_{R}(\Lambda)\right) \rightarrow \underline{K}_{0}\left(\underline{\mathrm{CM}}_{S}\left(\Lambda^{\prime}\right)\right)$ by $\tilde{\alpha}([A])=[\alpha(A)]$ for $A$ in $\mathrm{CM}_{R}(\Lambda)$. Next, we want to show that $\tilde{\alpha}$ is well defined. It is enough to show that $\tilde{\alpha}$ applied to any generator for the relations in $\underline{K}_{0}\left(\underline{\mathrm{CM}}_{R}(\Lambda)\right)$, is zero in $\underline{K}_{0}\left(\underline{\mathrm{CM}}_{S}\left(\Lambda^{\prime}\right)\right)$. Let $0 \rightarrow A \stackrel{f}{\rightarrow} B \oplus P \stackrel{g}{\rightarrow} C \rightarrow 0$ be an exact sequence in $\mathrm{CM}_{R}(\Lambda)$ with no split exact summands, where $A, B$, and $C$ have no nonzero projective summands in $\mathrm{CM}_{R}(\Lambda), A$ is indecomposable, and $P$ is projective in $\mathrm{CM}_{R}(\Lambda)$. If $f \neq 0$ in $\operatorname{Hom}_{\Lambda}(A, B)$, then Proposition 3 implies that there exists an exact sequence $0 \rightarrow \alpha(A) \rightarrow \alpha(B) \oplus Q \rightarrow \alpha(C) \rightarrow 0$ in $\mathrm{CM}_{S}\left(\Lambda^{\prime}\right)$ with $Q$ projective. Hence, $\tilde{\alpha}([A]+[C]-[B])$ is zero in $\underline{K}_{0}\left(\underline{\mathrm{CM}}_{S}\left(\Lambda^{\prime}\right)\right)$. If $\underline{f}=0$, then it follows from Theorem 2 that $0 \rightarrow(, \underline{B}) \stackrel{(, g}{\rightarrow}(, \underline{C}) \rightarrow \operatorname{coker}(, \underline{g}) \rightarrow 0$ is a minimal projective resolution of coker $(, \underline{g})$ in $\bmod \mathrm{CM}_{R}(\Lambda)$. Since the resolution is minimal and $(, \underline{B})$ is injective in $\bmod \underline{\mathrm{CM}}_{R}(\Lambda), B=(0)$ and our original sequence is of the form $0 \rightarrow \Omega_{\Lambda} C \rightarrow P \rightarrow C \rightarrow 0$, which gives the relation $[C]+\left[\Omega_{\Lambda} C\right]$ in $\underline{K}_{0}\left(\underline{C M}_{R}(\Lambda)\right)$. Then $\tilde{\alpha}\left([C]+\left[\Omega_{\Lambda} C\right]\right)=[\alpha(C)]+\left[\alpha \circ \Omega_{\Lambda} C\right]=$ $[\alpha(C)]+\left[\Omega_{\Lambda^{\prime}}(\alpha(C))\right]$, which is 0 in $\underline{K}_{0}\left(\underline{\mathrm{CM}}_{S}\left(\Lambda^{\prime}\right)\right)$, so that $\tilde{\alpha}$ is a well-defined homomorphism.

Since $\alpha$ is an equivalence, $\tilde{\alpha}$ is obviously onto. Now, we use the inverse, $\alpha^{-1}$, of $\alpha$ to define a map $\widetilde{\alpha^{-1}}: \underline{K}_{0}\left(\underline{\mathrm{CM}}_{S}\left(\Lambda^{\prime}\right)\right) \rightarrow \underline{K}_{0}\left(\underline{\mathrm{CM}}_{R}(\Lambda)\right)$, which will be an inverse of $\tilde{\alpha}$. Hence, $\tilde{\alpha}$ is an isomorphism.

Corollary 6. Let $\Lambda$ and $\Lambda^{\prime}$ be selfinjective artinian algebras over $R$ and $S$ respectively, where $R$ and $S$ are commutative local (Gorenstein) rings. If $\Lambda$ has no ring summand of Loewy length 2 and the categories $\bmod \Lambda$ and $\bmod \Lambda^{\prime}$ are equivalent, then

$$
\underline{K}_{0}(\underline{\bmod } \Lambda) \simeq \underline{K}_{0}\left(\underline{\bmod } \Lambda^{\prime}\right) .
$$


Proof. Assume that $\alpha: \underline{\bmod } \Lambda \rightarrow \bmod \Lambda^{\prime}$ is an equivalence. It is shown in [4, Theorem 4.4 (b)] that if $\Lambda$ has no indecomposable ring summand of Loewy length 2 , then $\Omega_{\Lambda^{\prime}} \circ \alpha \simeq \alpha \circ \Omega_{\Lambda}$ on all indecomposable nonprojective $\Lambda$-modules.

\section{THE MAIN RESULT}

For a commutative Cohen-Macaulay ring $R$ let $\mathrm{CM}(R)$ denote the category of all finitely generated maximal Cohen-Macaulay modules over $R$ and $\underline{\mathrm{CM}}(R)$ the stable category of $\operatorname{CM}(R)$. Then we have the following results, where $\Omega_{R}$ denotes the first syzygy in $\mathrm{CM}(R)$.

Theorem 7. Let $R$ and $S$ be commutative complete noetherian local Gorenstein domains. Assume that $\mathrm{CM}(R)$ and $\mathrm{CM}(S)$ are stably equivalent by an equivalence $\alpha: \underline{\mathrm{CM}}(R) \rightarrow \underline{\mathrm{CM}}(S)$ such that $\Omega_{S} \circ \alpha \simeq \alpha \circ \Omega_{R}$ on objects. Then

$$
K_{0}(\bmod R) \simeq K_{0}(\bmod S) .
$$

Proof. As mentioned just after Proposition 3, there exists an exact sequence of groups

$$
\eta: 0 \rightarrow \mathbf{Z} \rightarrow K_{0}(\mathrm{CM}(R)) \rightarrow \underline{K}_{0}(\underline{\mathrm{CM}}(R)) \rightarrow 0
$$

and the sequence splits if $R$ is a domain [3, Chapter 3]. Since by Theorem 5 $\underline{K}_{0}(\underline{\mathrm{CM}}(R)) \simeq \underline{K}_{0}(\underline{\mathrm{CM}}(S))$ and $R$ and $S$ are domains, we have

$$
K_{0}(\bmod R) \simeq K_{0}(\mathrm{CM}(R)) \simeq K_{0}(\mathrm{CM}(S)) \simeq K_{0}(\bmod S) .
$$

Now we apply our results to hypersurface rings. Let $T=k\left[\left[z_{1}, \ldots, z_{r}\right]\right]$ be the formal power series ring in the indeterminates $z_{i}$, and $k$ an algebraically closed field of characteristic different from 2 . Let $f$ be a nonzero nonunit in $T$, $R=T /(f)$ and $S=T[[x, y]] /(f+x y)$. Then, $\mathrm{CM}(R)$ and $\mathrm{CM}(S)$ are stably equivalent and the equivalence $\alpha: \underline{\mathrm{CM}}(R) \rightarrow \underline{\mathrm{CM}}(S)$ satisfies $\Omega_{S} \circ \alpha \simeq \alpha \circ \Omega_{R}$ on objects [8].

Corollary 8. If $R=T /(f), S=T[[x, y]] /(f+x y)$ and $R$ is a domain, then

$$
K_{0}(\bmod R) \simeq K_{0}(\bmod S) .
$$

As mentioned in the introduction it is known that if $R$ and $S$ are as in the above corollary and if $R$ is a domain of finite Cohen-Macaulay type, then $K_{0}(\bmod R) \simeq K_{0}(\bmod S)$. The computations of these groups for all hypersurface rings of finite Cohen-Macaulay type are given in [6]. We will end this paper with an example showing that one cannot remove the assumption that both rings are domains from Theorem 7 and Corollary 8 .

Example. Let $T=k\left[\left[z_{1}, z_{2}\right]\right]$ be the formal power series ring over an algebraically closed field $k$ of characteristic different from 2 in the indeterminates $z_{1}$ and $z_{2}, f=z_{1}^{2}$ and $R=T /(f)$. Then $f$ is the $A_{\infty}$ singularity in dimension 1 and all the indecomposable Cohen-Macaulay modules over $R$ are explicitly known [7]. They are $R, M_{0}=T /\left(z_{1}\right), M_{r}=T^{2} / \operatorname{Im} \varphi_{r}$, where 
$\varphi_{r}=\left(\begin{array}{cc}z_{1} & z_{2}^{r} \\ 0 & -z_{1}\end{array}\right): T^{2} \rightarrow T^{2}$ for natural numbers $r \geq 1$. It is easy to find the following exact sequences in $\mathrm{CM}(R)$

$$
0 \rightarrow M_{0} \rightarrow R \rightarrow M_{0} \rightarrow 0
$$

and

$$
0 \rightarrow M_{0} \rightarrow M_{r} \rightarrow M_{0} \rightarrow 0,
$$

so that $K_{0}(\bmod R)$ is generated by one element $\left[M_{0}\right]$.

If $R^{\prime}=k\left[\left[z_{1}\right]\right] /(f)$, then $R=R^{\prime}\left[\left[z_{2}\right]\right]$. Since $R$ is a flat $R^{\prime}$-module and $z_{2}$ is a nonzero divisor for all the Cohen-Macaulay modules over $R$, the functors $F=R \otimes_{R^{\prime}}: \mathrm{CM}\left(R^{\prime}\right) \rightarrow \mathrm{CM}(R)$ and $G=R /\left(z_{2}\right) \otimes_{R}-: \mathrm{CM}(R) \rightarrow \mathrm{CM}\left(R^{\prime}\right)$ are exact functors, and the composition $G \circ F$ is isomorphic to the identity functor on $\mathrm{CM}\left(R^{\prime}\right)$. Hence, $K_{0}\left(\bmod R^{\prime}\right)$ is a summand of $K_{0}(\bmod R)$ and, since $R^{\prime}$ is an artinian local ring, $K_{0}\left(\bmod R^{\prime}\right)=\mathbf{Z}$. Therefore $K_{0}(\bmod R)=\mathbf{Z}$, since $K_{0}(\bmod R)$ is a factor of $\mathbf{Z}$ and $\mathbf{Z}$ is a summand of $K_{0}(\bmod R)$.

Let $S=T[[x, y]] /\left(z_{1}^{2}+x y\right)$, which is the $A_{\infty}$ singularity in dimension 3 . Using that $\mathrm{CM}(R)$ and $\mathrm{CM}(S)$ are stably equivalent [8].we can find all the indecomposable Cohen-Macaulay modules over $S$ and they are

and

$$
S, M_{0}=(T[[x, y]])^{2} / \operatorname{Im}\left(\begin{array}{cc}
x & z_{1} \\
z_{1} & -y
\end{array}\right)
$$

for $r \geq 1$.

$$
M_{r}=(T[[x, y]])^{4} / \operatorname{Im}\left(\begin{array}{cccc}
x & 0 & z_{1} & z_{2}^{r} \\
0 & x & 0 & -z_{1} \\
z_{1} & z_{2}^{r} & -y & 0 \\
0 & -z_{1} & 0 & -y
\end{array}\right)
$$

As above, it is easy to find the following exact sequences in $\operatorname{CM}(S)$

$$
0 \rightarrow M_{0} \rightarrow S^{2} \rightarrow M_{0} \rightarrow 0
$$

and

$$
0 \rightarrow M_{0} \rightarrow M_{r} \rightarrow M_{0} \rightarrow 0
$$

so that $K_{0}(\bmod S)$ is a factor of $\mathbf{Z} \oplus \mathbf{Z}_{2}$.

If $S^{\prime}=k\left[\left[z_{1}, x, y\right]\right] /\left(z_{1}^{2}+x y\right)$, then $S^{\prime}$ is of finite Cohen-Macaulay type ( $A_{1}$ singularity) and we can use the stable AR-quiver for the category $\operatorname{CM}\left(S^{\prime}\right)$ to calculate $K_{0}\left(\bmod S^{\prime}\right)=\mathbf{Z} \oplus \mathbf{Z}_{2}$. Similarly, as above, $K_{0}\left(\bmod S^{\prime}\right)$ is a sum$\operatorname{mand}$ of $K_{0}(\bmod S)$ and, therefore, $K_{0}(\bmod S)=\mathbf{Z} \oplus \mathbf{Z}_{2}$. So, $K_{0}(\bmod R) \not$ $K_{0}(\bmod S)$ and, therefore, one cannot remove the assumption that both rings are domains from Theorem 7 and Corollary 8.

\section{ACKNOWLEDGMENTS}

This work was started together with Alex Martsinkowski by studying the hypersurface case and I thank him for sharing his insight and ideas with me on examples and trying out different methods of proofs. I also thank Idun Reiten for helpful comments. 


\section{REFERENCES}

1. M. Auslander, Functors and morphisms determined by objects, Proc. Conf. Representation Theory (Philadelphia, 1976), Marcel Dekker, New York, 1978, pp. 1-244.

2. __ Isolated singularities and existence of almost split sequences, Representation Theory II, Lecture Notes in Math., vol. 1178, Springer-Verlag, New York, 1986, pp. 194-241.

3. M. Auslander and I. Reiten, Grothendieck groups of algebras and orders, J. Pure Appl. Algebra 39 (1986), 1-51.

4. __ Representation theory of artin algebras VI: A functorial approach to almost split sequences, Comm. Algebra 11 (1977), 257-300.

5. _ Stable equivalence of artin algebras, Proc. Conf. on Orders, Group Rings and Related Topics, Lecture Notes in Math., vol. 353, Springer-Verlag, 1972, pp. 8-71.

6. J. Herzog and H. Sanders, The Grothendieck groups of invariant rings and of simple hypersurface singularities, Proc. of Conf. on Singularities, Representation of Algebras and Vector Bundles, Lecture Notes in Math., vol. 1273, Springer-Verlag, 1987, pp. 134-149.

7. R.-O. Buchweitz, G.-M. Greuel, and F. O. Shreyer, Cohen-Macaulay modules on hypersurface singularities. II, Invent. Math. 88 (1987), 165-182.

8. H. Knörrer, Cohen-Macaulay modules on hypersurface singularities. I, Invent. Math. 88 (1987), 153-164.

Institutt for Matematikx og Statistikk, Universitetet i Trondheim, AVH, N-7055 DRAGVOLL, NORWAY 